\title{
Pyoderma gangrenosum-A case report
}

\begin{abstract}
A 36 year old lady presented to Mayo Hospital Lahore, Pakistan with a 6 month history of widespread, discrete ulcer formation all over her body. The ulcers started from her left hand, as an itchy erythematous rash. The overlying skin broke down within 2-3 days to form an ulcer. The patient developed several such ulcers on the rest of her body involving her arms, legs, buttocks, breasts and abdomen. The ulcers were painful, non-healing and unsightly, restricting her daily activities and leading to psychosocial stress. She had previously received treatment consisting of corticosteroids, antibiotics and was started on cyclosporine 1 month before presentation. Although partial relief was found with no further ulcer formation, the pre-existing ulcers were extremely slow to heal. There was no complete symptom free period during the 6 months of her illness.
\end{abstract}

Volume 4 Issue 3 - 2020

\author{
Mahym Mansoor, Ghazala Butt,Amna Asad, \\ ljaz Hussain \\ Department of dermatology, Mayo Hospital KEMU, Pakistan
}

Correspondence: Ghazala Butt, Department of dermatology, Mayo Hospital, King Edward Medical University, Pakistan, Email ghazalakashiri@gmail.com

Received: May 27, 2020 | Published: June 04, 2020

\section{Case report}

Prior to this, patient had a history of miscarriage at 3rd month of pregnancy 6 months back, overlapping with the disease onset. Patient also suffered from renal colic secondary to an $11 \mathrm{~mm}$ right renal stone for which she multiple I/V and I/M injections of nalbuphine at the start of ulcer formation. Her disease recurred at sites of canula insertion, leading to further painful ulcer formation, necessitating further parenteral administration of painkillers, leading to a vicious cycle of disease aggravation. Patient also gave history of weight gain of $20 \mathrm{~kg}$ over the last 6-7 months, along with a husky character of voice for the last month or so along with a sore mouth and odynophagia. There was no history of change in bowel movements, melena or hematochezia. Family history was insignificant and she was a mother of 3. On general examination, she was markedly pale, obese (BMI 37.5) with a husky voice and a Cushingoid face. She was hemodynamically stable. Ambulation was difficult due to movement restricting painful ulcers. Multiple ulcers were present on both arms, legs, buttocks, and breasts, size ranging from $1-10 \mathrm{~cm}$ in diameter with regular margins, undermined edges with violaceous border, tender to palpation, the floor was covered with necrotic slough and eschar, and the base was formed by muscles and subcutaneous fat. Some of the ulcers had a foul-smelling purulent discharge.

Routine blood count showed a hemoglobin of $5 \mathrm{~g} / \mathrm{dl}$ with $\mathrm{MCV}$ of $99 \mathrm{fl}$ and a retic count of $11 \%$. Serum Bilirubin levels, LDH levels and haptoglobin levels were normal. Direct and Indirect Coomb's test, ANA and ENA profile were negative. Stool for occult blood was positive so patient underwent both upper and lower GI endoscopy. The upper GI series showed mild hyperemia of gastric mucosa secondary to NSAID/corticosteroid intake. Colonoscopy showed

Thyroid function tests were also done which revealed a raised TSH level of $4.72 \mathrm{mIU} / \mathrm{L}$ (N 0.25-4.00), free T4 5.00pmol/ (N 9.00-23.2), free T3 $1.61 \mathrm{pmol} / \mathrm{L}$ (N 2.8-6.5). Anti-thyroid peroxidase antibodies, thyroid scan. She was also found to be diabetic with a fasting glucose levels of $246 \mathrm{mg} / \mathrm{dl}$ and random $378 \mathrm{mg} / \mathrm{dl}$. HbAlc was 8.1 . Rest of the liver and renal profile were within normal limits. RA factor was 15IU/ $\mathrm{mL}(\mathrm{ULN}<14)$ but there was no associated joint pain. HBsAg and Anti-HCV were negative. CXR showed left sided pleural effusion. Biopsy was done which showed an ulcer with a necrotic base and neutrophilic crusts, surrounded by normal epidermis. There was a dense neutrophilic infiltrate in the dermis. A medium sized vessel in the mid dermis showed fibrinoid degeneration and intramural and perivascular neutrophilic infiltrate. No evidence of malignancy was found.

Patient was admitted with the provisional diagnosis of ulcerative type of pyodermagangrenosum. Swab for wound culture/sensitivity were taken which grew out Citrobacterand Proteus species sensitive to amikacin. She was started on inj. Dexamethasone $8 \mathrm{mgI} / \mathrm{V} /$ day along with Cyclosporine 100mg twice daily. Inj. Amikacin 500mg thrice daily was also given for 14 days. Tab. Thyroxine 100ug/day was also initiated along with insulin during the hospital stay. 3 packed cell transfusions were also given to the patient. Meticulous wound care along with surgical debridement of necrotic eschars was done.

Patient improved dramatically after starting thyroxine and controlling blood sugar levels. Some of the smaller ulcers healed completely with cribriform scarring. The quality of voice also improved after initiation of thyroxine. Her pleural effusion also resolved after starting thyroxine. Retic count decreased to 3.6\% after PPI's were started and her hemoglobin slowly built up to $10 \mathrm{mg} / \mathrm{dl}$.

\section{Discussion}

Pyodermagangrenosum is a rare, non-infectious, autoimmune neutrophilicdermatosis. ${ }^{1}$ It is more common in females with a median age of 59 years. $^{2}$ Clinically, it has several variants but the classical ulcerative type is the commonest. ${ }^{2}$ It presents as small, tender, violaceous papules which evolve into ulcers with undermined edges and a violaceous border. It can involve any part of the body and the lesions may be multiple. It heals with classical cribriform scarring. Histopathological findings include central necrosis and ulceration of epidermis and dermis with an intense neutrophilic infiltrate, but it is not diagnostic and is usually undertaken to exclude other causes. ${ }^{3}$ Its treatment is complex and depends upon the extent of the lesions. Wound care, corticosteroids, ciclosporin, methotrexate, dapsone, cyclophosphamide, azathioprine and biologics such as infliximab and etanercept are just a few of the modalities and treatment options used. ${ }^{2}$ The treatment is often prolonged and therapy is tailored for each individual patient.

Severalstudieshavedescribedalinkbetweenpyodermagangrenosum and other systemic diseases such as Inflammatory Bowel Disease, rheumatoid arthritis, hematological malignancies/monoclonal gammopathy and vasculitides. These underlying disease states 
had a 4-6 fold increase in in-hospital mortality as well as resource utilization, thus enforcing the need to effectively screen for underlying systemic disease states. ${ }^{4}$ Historically, IBD was reported to be the most common associated disease ${ }^{5}(32 \%){ }^{6}$ Recently, focus has been shifted on detecting underlying endocrine disorders as several studies have found a potential link with diabetes mellitus, thyroid disorders and metabolic syndrome. A multi-centric analysis conducted in 259 patients in Germany revealed that patients of pyodermagangrenosum had concomitant anemia in $45.6 \%$ of the patient population, $44.8 \%$ had endocrine disease, $12.4 \%$ had internal malignancies, and $9.3 \%$ had chronic inflammatory bowel disease. $25.5 \%$ of the patients had diabetes mellitus with some aspects of metabolic syndrome. ${ }^{7}$ Another study found endocrine disease in $38.8 \%$ of the patients, diabetes in $28.6 \%$ of the patients and obesity in $32.6 \%{ }^{8}$ An association of antithyoid drug propylthiouracil has been described in literature. ${ }^{9}$ Several case reports have described favourable outcome after treatment of associated endocrine abnormalities. ${ }^{10-12}$

The patient in our report had been on corticosteroids as well as ciclosporin for several months without an adequate response before her hypothyroidism and diabetes were diagnosed. Institution of thyroxine as well as control of her blood sugar levels dramatically accelerated the healing of her ulcers. She was continued on oral corticosteroids and ciclosporin with wound care and was advised to lose weight (Figure 1-2).

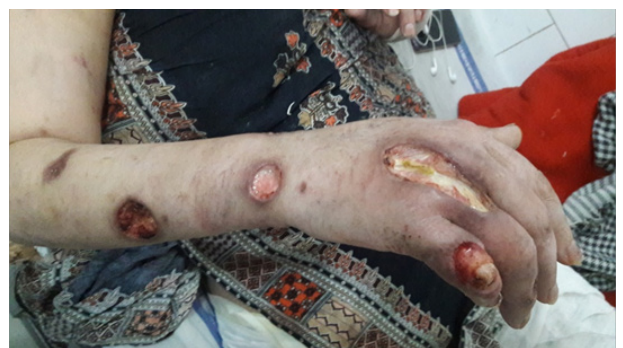

Figure I Punched out deep ulcers seen on right forearm and hand of the patient.

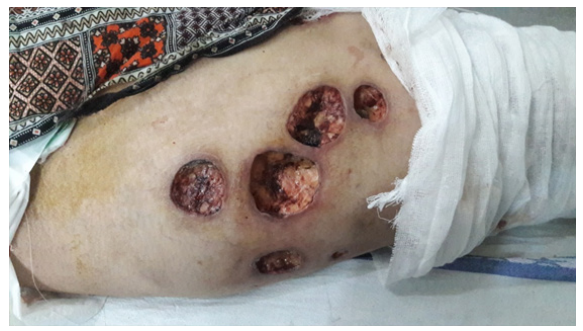

Figure 2 Circular shaped punched out ulcers of Pyodermagangrenosum.

\section{Conclusion}

Pyodermagangrenosum is an uncommon, sterile, neutrophilic dermatoses which leads to chronic ulceration. It has well-known associations with other systemic diseases which may precede, concur or follow its course. Endocrine diseases associated with pyodermagangrenosum have recently come into the limelight as several studies have suggested a close association, hence patients must be screened for any underlying diabetes or thyroid disorder. The concomitant management of these diseases may achieve remission or a favourable outcome with less need of other immunosuppressive agents, and also improve the quality of life for these patients.

\section{Conflicts of interest}

The author declares that there is no conflicts of interests.

\section{Acknowledgments}

None.

\section{Funding}

None.

\section{References}

1. Ruocco E, Sangiuliano S, Gravina AG, et al. Pyodermagangrenosum: an updated review. J Eur Acad Dermatol Venerol. 2009;23(9):1008-1017.

2. Omerod AD, Hampton PJ. Neutrophilic Dermatoses. In: Griffiths C, Barker J, editors. Rook's Textbook of Dermatology. 9th ed. Hoboken, NJ; Blackwell Publishing. 2016;(2):49-1.

3. Crowson AN, Mihm MC Jr, Magro C. Pyodermagangrenosum: a review. $J$ Cutan Pathol. 2010;30(2):97-107.

4. Kaffenberger BH, Hinton A, Krishna SG. The impact of underlying disease state on outcomes in patients with pyodermagangrenosum: A national survey. J Am Acad Dermatol. 2018;79(4):659-63.

5. Suárez-Pérez JA, Herrera-Acosta E, López-Navarro $\mathrm{N}$, et al. Pyodermagangrenosum: a report of 15 cases and review of the literature. Actas Dermosifiliogr. 2012;103(2):120-126.

6. Vacas AS, Torre AC, Bollea-Garlatti ML, Warley F, et al. Pyodermagangrenosum: clinical characteristics, associated diseases, and responses to treatment in a retrospective cohort study of 31 patients. Int $J$ Dermatol. 2017;56(4):386-391.

7. Al Ghazal P, Herberger K, Schaller J, et al. Associated factors and comorbidities in patients with pyodermagangrenosum in Germany: a retrospective multicentric analysis in 259 patients. Orphanet $J$ Rare Dis. 2013;8:136.

8. Al Ghazal P, Körber A, Klode J, et al. Investigation of new co-factors in 49 patients with pyodermagangrenosum. J Dtsch Dermatol Ges. 2012;10(4):251-257.

9. Seo JW, Son HH, Choi JH, et al. A case of p-ANCA-positive propylthiouracil-induced pyodermagangrenosum. Ann Dermatol. 2010;22(1):48-50.

10. Necas M, Semrádova V, Vaskù V. Pyoderma gangraenosum associated with autoimmune thyreopathy and hyperandrogenic syndrome. Acta Dermatovenerol Alp Pannonica Adriat. 2005;14(2):57-60.

11. Agarwal US, Agarwal P, Prakash C, et al. Ulcerative Pyoderma Gangrenosum and Leukocytoclastic Vasculitis in a Hypothyroid Woman: A Case Report. Wounds. 2017;29(6):43-47.

12. Oumakhir S, Frikh R, Ghfir M, et al. A case of pyodermagangrenosum of favourable outcome after treatment of associated hyperthyroidism. Ann DermatolVenereol. 2009;136(4):367-368. 